\title{
Qualche osservazione su alcuni generali procedimenti di sommazione delle serie (*).
}

\author{
Memoria di Carlo Birinderliti (a Roma).
}

A Mauro Picone nel suo $70^{\text {mo }}$ compleanno

Sunto. - A mezzo di alcune opportune osservazioni si mette in rilievo la possibilità di realizzare dei perfezionamenti in assai generali procedimenti di sommazione delle serie. Inoltre, con qualche opportuno impiego e iterazione di semplici algoritmi, si espongono vari procedimenti di sommazione semplici nella struttura e utili nell' impiego per la risoluzione di problemi inerenti al prolungamento analitico.

1. È noto il seguente procedimento $\left({ }^{1}\right)$ assai generale per la sommazione delle serie.

In un insieme illimita to $A$ dello spazio $S_{(r)}$ sia definita una successione di funzioni $f_{k}(P)$ reali, per $k=0,1,2, \ldots$, sempre limitate ma non negative tali che

$$
\begin{aligned}
f_{0}(P) & \geq f_{1}(P) \geq \ldots \geq f_{k}(P) \geq \ldots, \\
& \lim _{P \rightarrow \infty} f_{k}(P)(\text { su } A)=1,
\end{aligned} \quad k=0,1.2, \ldots .
$$

Assegnata una serie $\stackrel{0, \infty}{\Sigma_{n}} u_{n}$, si ponga, qualunque sia il $P$ scelto in $A$,

$$
F_{n}(P)=\stackrel{0, n}{\Sigma_{k}} f_{k}(P) \cdot u_{k}, \quad \begin{aligned}
& \lim _{n \rightarrow \infty}^{\prime} F_{n}(P)=F^{\prime}(P), \\
& \lim _{n \rightarrow \infty} F_{n}(P)=F^{\prime \prime}(P),
\end{aligned}
$$

per definire poi come minima e massima somma, generalizzata, su $A$, della serie $\Sigma_{n}^{0, \infty} u_{n}$ e relativamente alla successione (1) i seguenti limiti

$$
\left.\lim _{P \rightarrow \infty} F^{\prime}(P) \text { (su } A\right), \quad \lim _{P \rightarrow \infty} F^{\prime \prime}(P) \text { (su } A \text { ); }
$$

In tal senso $\mathrm{s}^{\prime}$ intende che la serie risulta sommabile o non sommabile, su $A$ e relativamente alla successione (1), se tali limiti sono oppur no coincidenti.

Nel primo caso il valore comune di tali limiti da la somma, su $A$, generalizzata della serie ${ }^{0, \infty} u_{n}$ relativa alla successione (1) impiegata (ciò avviene

(*) Lavoro eseguito presso l'Istituto Nazionale per le Applicazioni del Calcolo.

(i) Mauro Picone, Sui metodi di sommazione delle serie, Annali di Matematica pura ed applicata», serie IV, tomo II (1924-1925), Zanichelli, Bologna. 
solo se le funzioni $F^{\prime \prime}(P), F^{\prime \prime}(P)$ sono entrambe regolari, su $A$, all'infinito, possedendo inoltre un limite comune).

Secondo che tale limite comune è finito od infinito la serie è convergente o divergente relativamente al procedimento di sommazione generalizzata impiegato.

Si noti il perfezionamento che l'uso di tali procedimenti comporta. a confronto degli altri metodi di sommazione delle serie, col non prendere affatto in considerazione la questione della convergenza ordinaria della serie $\stackrel{0, \infty}{\Sigma_{n}} f_{n}(P) \cdot u_{n}$.

Il risultato di fondamentale rilievo è poi fornito dal seguente

Teorema. - L'intervallo di indeterminazione del procedimento di somma-

zione di una serie $\sum_{n}^{0, \infty} u_{n}$, su qualsiasi insieme $A$ e relativo ad una qualsiasi successione (1), è sempre contenuto nell'intervallo di indeterminazione del pro. cedimento di sommazione ordinaria della serie $\Sigma_{n} u_{n}$.

2. Modificando leggermente le modalità di impiego del procedimento è agevole rendersi conto che è possibile ottenere dei perfezionamenti riguardo al risultato indicato nel teorema del n. 1.

Si pensi ad esempio una volta prefissato un punto $P$ di $A$ di prendere in considerazione la totalita dei punti $Q$ di $A$ aventi dalla origine $O$ (dello spazio $S_{(r)}$, cui appartiene $A$ ) distanza non minore di $O P$ e di indicare con $A_{P}$ tale insieme, di punti $Q$, sicchè, qualunque sia il punto $P$ di $A$ risulta sempre $A_{P} \subset A$.

太 chiaro allora che si può prendere in considerazione in luogo dell'ordinario polinomio $F_{n}(P)=\stackrel{0 . n}{\Sigma_{k}} u_{k} f_{k}(P)$ l'altro analogo $F_{n}(P, Q)=\stackrel{N}{*}, n_{\Sigma_{k}} u_{k} \cdot f_{k}(P) \cdot f_{k}(Q)$ e ricercare anzitutto il minimo ed il massimo limite (tenendo fisso $P$ di $A$ e un $Q$ arbitrario di $\left.A_{P}\right)$ per $n \rightarrow \infty$

$$
F^{\prime}(P, Q)=\lim _{n \rightarrow \infty} F_{n}(P, Q), \quad F^{\prime \prime}(P, Q)=\lim _{n \rightarrow \infty} F_{n}(P, Q)
$$

e poi i limiti

$$
\Psi(P)=\lim _{Q \rightarrow \infty} F^{\prime}(P, Q)\left(\text { su } A_{P}\right), \quad \Phi(P)=\lim _{Q \rightarrow \infty} F^{\prime \prime}(P, Q)\left(\text { su } A_{P}\right)
$$

che vengono a costituire la minima somma e la massima somma della serie $\stackrel{0}{\Sigma}, \infty_{n} u_{n} \cdot f_{n}(P)$ e relative al procedimento di sommazione su l'insieme $A_{P}$ di punti $Q$ e con la successione $f_{k}(Q)$. Per il teorema del n. 1 , si ha che la minima somma ordinaria e la massima somma ordinaria della serie $\stackrel{0, \infty}{\Sigma_{n}} u_{n}$. - $f_{n}(P)$ comprendono tra di loro quelle generalizzate $\Psi(P), \Phi(P)$ or ora dedotite per la stessa serie, vale a dire è

$$
F^{\prime}(P) \leq \Psi(P) \leq \Phi(P) \leq F^{\prime \prime}(P)
$$


Con un ultimo passaggio al limite, per $P \rightarrow \infty$ su $A$, si deduce dalla (5) ch' ̀̀ (6) $\lim _{P \rightarrow \infty} F^{\prime}(P)($ su $A) \leq \lim _{P \rightarrow \infty} \Psi(P)(\operatorname{su} A) \leq \lim _{P \rightarrow \infty} \Phi(P)($ su $A) \leq \lim _{P \rightarrow \infty} F^{\prime \prime}(P)(\operatorname{su} A)$, sicchè definendo per minima somma e massima somma della serie $\Sigma_{n}^{0 . \infty} u_{n}$, ottenute col metodo espresso in questo n., i due

$$
\lim _{P \rightarrow \infty} \Psi(P) \text { (su } A \text { ), } \lim _{P \rightarrow \infty} \Phi(P) \text { (su } A \text { ), }
$$

si può affermare che col nuovo procedimento di sommazione per una serie ${ }_{\Sigma_{n}}^{0, \infty} u_{n}$ il competente intervallo di indeterminazione $\dot{e}$ contenuto in quello relativo al procedimento di sommazione della serie stessa su l'insieme $A$ e relativo alla successione (1).

Ciò costituisce un perfezionamento del risultato espresso dal teorema indicato al n. 1.

3. Il metodo di ABEL-Porsson rientra fra quelli della natura precisata al n. 1. Basta pensare di prendere in considerazione come successione (1) una successione

$$
1, \Theta(P),[\Theta(P)]^{2},[\Theta(P)]^{3}, \ldots,[\Theta(P)]^{n}, \ldots,
$$

ove per ogni $P$ di $A$ risulti $0<\theta(P)<1$ con

$$
\lim _{P \rightarrow \infty} \Theta(P)(\mathrm{su} A)=1
$$

Posto quindi, per ogni punto $P$ di $A$, in relazione ad una serie ${ }^{0, \infty} \Sigma_{n} u_{n}$,

$$
\begin{gathered}
A_{n}(P)=u_{0}+u_{1} \cdot \Theta(P)+u_{2} \cdot[\Theta(P)]^{2}+\ldots+u_{n} \cdot[\Theta(P)]^{n}+\ldots, \\
\lim _{n \rightarrow \infty} A_{n}(P)=A^{\prime}(P) ; \quad \lim _{n \rightarrow \infty}^{\prime \prime} A_{n}(P)=A^{\prime \prime}(P),
\end{gathered}
$$

si diranno minima e massima somma su $A$, secondo il metodo ABEL-PoIsson e in relazione alla suecessione di potenze $\left\{[\Theta(P)]^{n}\right\}$, i limiti

$$
\lim _{P \rightarrow \infty}^{\prime} A^{\prime}(P) \text { (su } A \text { ), } \quad \lim _{P \rightarrow \infty}^{\prime \prime} A^{\prime \prime}(P) \text { (su } A \text { ). }
$$

4. Il perfezionamento che si può dare a tal metodo di ABEL-Porsson, operando nel modo seguito al $\mathrm{n}$. $2 \mathrm{nel}$ caso generale d' una qualsiasi succes. sione'(1), si realizza ovviamente come segue:

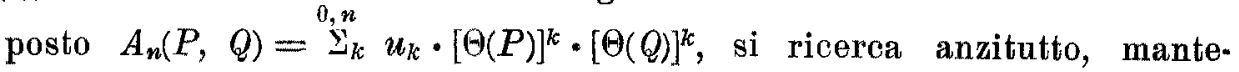
nendo fisso un $P$ di $A$ e un $Q$ arbitrario scelto comunque in $A_{P}$,

e poi i

$$
A^{\prime}(P, Q)=\lim _{n \rightarrow \infty} A_{n}(P, Q), \quad A^{\prime \prime}(P, Q)=\lim _{n \rightarrow \infty} A_{n}(P, Q),
$$

$$
\Psi(P)=\lim _{Q \rightarrow \infty}^{\prime} A^{\prime}(P, Q)\left(\mathrm{su} A_{P}\right), \quad \mathrm{X}(P)=\lim _{Q \rightarrow \infty} A^{\prime \prime}(P, Q)\left(\mathrm{su} A_{P}\right),
$$


onde definire come minima e massima somma generalizzate della serie ${ }^{0, \infty} u_{n}$ (ottenute col perfezionamento del metodo di ABEL-Poisson del n. 3) i due limiti seguenti

$$
\left.\lim _{P \rightarrow \infty} \Psi(P) \text { (su } A \text { ), } \quad \lim _{P \rightarrow \infty} \mathrm{X}(P) \text { (su } A\right),
$$

e il relativo intervallo di indeterminazione è contenuto in quello relativo al metodo di Abel-Poisson.

Osservazrone. - Operando come si è fatto al n. 2 ed al n. 4, o con analoghe modalità, e servendosi sempre solo della fondamentale successione delle assegnate $f_{k}(P)$, si può contrarre sempre più l'intervallo di indeterminazione, per una serie, quando l'applicazione dei metodi perfezionati venga iterata più volte.

5. Assegnato un arbitrario procedimento lineare di ToEpuITz, sia $U_{n}=\Sigma_{\nu}^{0, n} a_{n, \nu} \cdot u_{\nu}$ il polinomio $n^{0}$ di approssimazione per una serie $\Sigma_{\nu}^{0, \infty} u_{\nu}$. Tale procedimento lineare di Toepuitz ( $\left.{ }^{2}\right)$ rientra nel caso dei procedimenti del n. 1 se la successione, con numero finito di elementi,

$$
a_{n, 0}, a_{n, 1}, a_{n, 2}, \ldots, a_{n, n}, \quad\left(\text { per cui ciò̀ } a_{n, \nu}=0 \text { per } \vee>n\right. \text { ), }
$$

ha tutti gli elementi reali non negativi e se non è crescente $\left({ }^{2}\right)$. Sotto tali con. dizioni si ha dunque ohe l'intervallo di indeterninazione per il procedimento di Tosplitz e relativo alla sommazione d'una serie $\stackrel{0, \infty}{\nu_{y}} u_{v}$ è sempre contenuto in quello relativo al procedimento d'ordinaria sommazione della serie stessa.

Senza entrare nei minimi dettagli di talj metodi in cui il polinomio $n^{0}$ di approssimazione per una serie $\sum_{y}^{0, \infty} u_{\text {, }}$ appare sotto forma di combinazione lineare dei termini $u_{0}, u_{1}, \ldots, u_{n}$ mediante coefficenti, dipendenti solo da $n$ e $\nu, a_{n, \nu}$ reali non negativi e non crescenti al crescere di $\nu$, ci riferiremo qui, d'ora innanzi, solo a quelli, d'altronde svariatissimi, che applicati per la sommazione generalizzata della serie geometrica $\Sigma_{y}^{0, \infty} z^{y}$ danno come somma generalizzata

$$
\lim _{n \rightarrow \infty} U_{n}(z)=\lim _{n \rightarrow \infty} \stackrel{0, n}{\Sigma_{y}} a_{n, \nu} \cdot z^{\nu}=\frac{1}{1-z}
$$

in tutto un campo $D$ semplicemente connesso del piano complesso $z$ cui appartenga tutto il dominio $|z| \leq 1$, eccetto il punto $z=1$ singolare per la funzione olomorfa $\frac{1}{1-z}$.

Poichè, per esser reali le $a_{n, \nu}$, i due numeri $U_{n}(z), U_{n}(\bar{z})$, sono complessi coniugati, avviene che se converge $U_{n}(z)$, per $n \rightarrow \infty$, altrettanto avviene per $U_{n}(\bar{z})$ e i due limiti sono complessi coniugati. Il campo $D$ risulta sempre per

(2) I cui coefficienti verifichino le note condizioni di Toeprıtz.

(2') Intendendo cioè che sia $a_{n, \nu} \geq a_{n, v+1}$. 
tal ragione siłmmetrico rispetto all'asse reale, sicchè se, come sempre qui supporremo, la frontiera $L$ di $D$ è una curva regolare semplice, tal curva $L$ è pure essa simmetrica rispetto all' asse reale e, contenendo internamente tutta la circonferenta $|z|=1$ (eccetto $z=1$ ), si chiude nel punto, in genere angoloso, $z=1$ ch'è il solo punto ch' essa viene ad avere in comune con la $|z|=1$.

È essenziale per il seguito l'ammettere che $L$ abbia in comune un solo punto con ciascuna semiretta uscente da $z=0$ e che la convergenza della successione $U_{n}(z)=\stackrel{0, n}{\Sigma_{y}} a_{n, v} \cdot z^{v}$ verso la funzione olomorfa $\frac{1}{1-z}$ avrenga uniformemente quando $z$ si mantiene in domini contenuti nel campo $D$.

Tale complesso di modalità è, ripetiamo, verificato dalla maggior parte dei namerosissimi procedimenti lineari di sommazione che per varie vie possonsi realizzare, sicchè le conseguenze che vogliamo qui trarre dalla loro supposta validita hanno carattere di notevole generalita col permettere in tutti i singoli casi di tali procedimenti lineari di dedurre famiglie d'infiniti procedimenti pure lineari ottenibili con l'impiego di sottogruppi via via più radi appartenenti tutti al fondamentale insiem $\Theta\left\{a_{n, \nu}\right\}$ si che la struttura di tali procedimenti lineari appare più semplice di quello $U_{n}$ da cui si parte e il contributo ch'essi possono portare, nel caso della loro applicazione al problema del prolungamento analitico d' un elemento di funzione olomorfa $f(z) \cong \Sigma_{\nu} u_{\nu} z^{\nu}$, appare spesso via via più cospicuo sia nel raffronto fra di essi

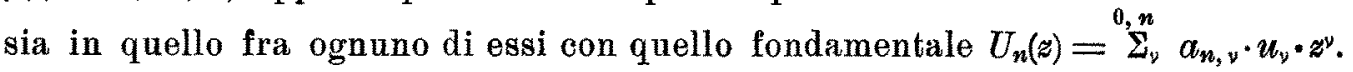

a) Si tenga presente che in genere, mentre nei punti del campo $D$ è $\lim _{n \rightarrow \infty} \sum_{\nu} \Sigma_{n, \nu} \cdot z^{\nu}=\frac{1}{1-z}$, se $z$ è scelto lungo la frontiera $L$ di $D$ o esternanamente a $D$ la successione $\Sigma_{y}^{0, n} a_{n, v} \cdot z^{\nu}$ non è convergente per $n \rightarrow \infty$.

Assunti valori di $n$ solamente pari si prenda in esame l'espressione seguente

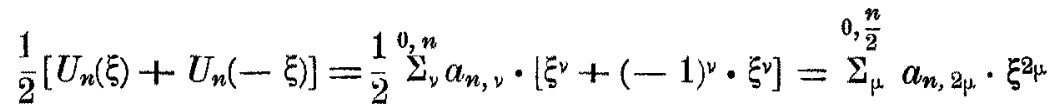

$$
0, \frac{n}{2}
$$

e si ósservi che tale polinomio $\Sigma_{\mu} a_{n, 2_{\mu}} \cdot \xi^{2 \mu}$ converge uniformemente, per $n \rightarrow \infty$, verso $\frac{1}{2}\left[\frac{1}{1-\xi}+\frac{1}{1+\xi}\right]=\frac{1}{1-\xi^{2}}$ in ogni dominio appartenente al campo $E$ costituito dall'insieme prodotto del campo $D$ e di quello ottenuto da $D$ col ribaltamento del piano complesso rispetto all'asse immaginario. La frontiera di $E$ è costituita evidentemente dalla parte di $L$ avente i punti di argomento compreso tra $-\frac{\pi}{2}$ e $\frac{\pi}{2}$ e della curva ottenuta da tale parte di $L$ col solito ribaltamento del piano complesso rispetto all'asse immaginario; 
tal curva $\mathcal{L}$, frontiera di $E$, racchiude internamente la circonferenza $|z|=1$ e ha comuni con questa $i$ soli due punti (angolosi) -1 e +1 .

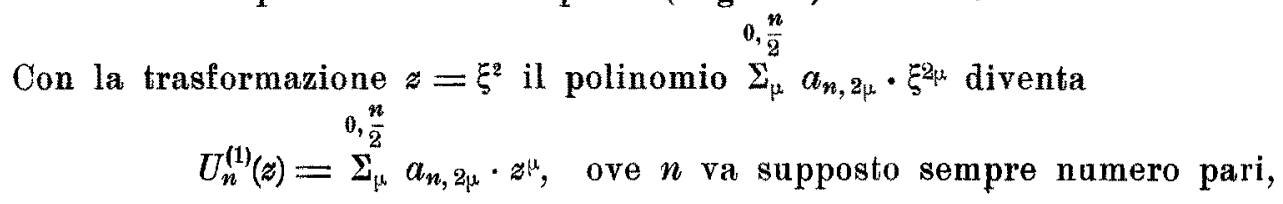

e il campo $E$ di punti $\xi$ si muta nel campo $E^{(1)}$ di punti $z$ limitato avente come frontiera la linea chiusa $L^{(1)}$ ottenuta trasformando mediante la $z=\xi^{2}$ la parte di $L$ costituita dai punti $\xi$ di $L$ aventi l'argomento compreso fra $-\frac{\pi}{2}$ e $\frac{\pi}{2}$.

Tal curva $L^{(1)}$ frontiera di $E^{(1)}$ ha $z=1$ e lo $z$ posto lungo il semiasse reale negativo in genere entrambi angolosi e racchiude internamente la circonferenza $|z|=1$ ma ha in comune con questa il punto $z=1$ e questo soltanto.

Il dominio ciroolare $|z| \leq 1$ è così contenuto nel eampo $E^{(1)}$ eccetto il punto $z=1$ che sta lungo la frontiera $L^{(1)}$ di $E^{(1)}$.

Da quanto si è fatto risulta che in tutto $E^{(1)}$ ò

$$
\lim _{n \rightarrow \infty} U_{n}^{(1)}(z)=\lim _{n \rightarrow \infty} \Sigma_{\mu}^{0, \frac{n}{2}} a_{n, 2 \mu} \cdot z^{\mu}=\frac{1}{1-z}
$$

e inoltre uniformemente in ogni dominio appartenente al campo $E^{(n)}$.

b) Se è assegnato un elemento $F(z) \simeq \Sigma_{\nu}^{0, \infty} u_{\nu} \cdot z^{\nu}$ di funzione olomorfa, ove il raggio del cerchio di convergenza è supposto diverso da zero, il poli nomio di approssimazione relativo al procedimento ora introdotto è ovviamente

$$
U_{n}^{(1)}(z)=\Sigma_{\mu}^{0, \frac{n}{2}} a_{n, 2 \mu} \cdot u_{\mu} \cdot z^{\mu} \text {. }
$$

Per quel che si è supposto riguardo ai caratteri della frontiera $L$ del campo $D$ segue che anche la frontiera $L^{(1)}$ di $E^{(1)}$ soddisfa necessariamente anch'essa alle solite modalità d'esser simmetrica rispetto all'asse reale e d'avere una sola intersezione con ogni semiretta uscente da $z=0$.

Con noto procedinento dimostrativo, detto $\alpha$ il primo punto singolare che si incontra quando partendo da 0 si fa il prolungamento analitico retti lineo dell' elemento assegnato $F(z) \simeq \Sigma_{\nu}^{0, \infty} u_{\nu} \cdot z^{\nu}$ di funzione olomorfa lungo qualsiasi semiretta spiccata da 0 , si trova che:

Se $E_{F}^{(1)}$ indica il campo parte comune a tutti $i$ campi $\alpha \cdot E^{(1)}$, in ogni punto $z$ del campo $E_{F}^{(1)} \grave{e}$

$$
\lim _{n \rightarrow \infty} U_{n}^{(1)}=\lim _{n \rightarrow \infty} \Sigma_{\mu}^{0, \frac{n}{2}} a_{n, 2 \mu} \cdot u_{\mu} \cdot z^{\mu}=F(z),
$$


ove $F(z)$ è il ramo monodromo di funzione olomorfa che si ottiene col prolungamento reltilineo dell'elemento ${ }^{0} \Sigma_{\nu}^{\infty} u_{v} z^{v}$ entro la relativa stella rettilinea di centro $z=0$; tale convergenza di $U_{n}^{\{1\}}$ a $F(z)$, per $n \rightarrow \infty$, è uniforme in ogni dominio $T$ contenuto in $E_{F}^{(1)}$, mentre d'altra parte il campo $E_{F}^{(1)}$ di sommabilità (di tal metodo $U_{n}^{(1)}$ verso la somma $F(z)$ per la serie ${ }^{0, \infty} u_{y} \cdot z^{y}$ ) sorpassa natu-

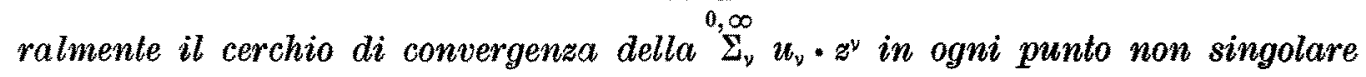
della circonferenza di convergenza.

$\left.a^{\prime}\right)$ Se il procedimento indicato in $a$ ) si itera $h$ volte complessivamente si ha che l'arbitrario $n$ va scelto fra $i$ multipli successivi di $2^{h}$ e per la serie geometrica $\Sigma_{v}^{0, \infty} z^{v}$ si trova quale polinomio d'approssimazione $U_{n}^{(h)}(z)$

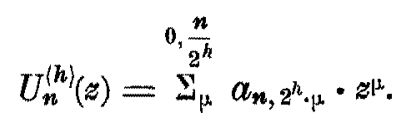

Presa la parte di $L$ costituita dai punti $\xi$ di $L$ aventi l'argomento compreso fra $-\frac{\pi}{2^{h}}, \frac{\pi}{2^{h}}$, si consideri la trasformata $L^{(h)}$ di essa ottenuta mediante la $z=\xi^{2^{h}}$. La curva $L^{(h)}$ è chiusa e simmetrica rispetto all' asse reale e in genere è dotata di due punti angolosi dei quali uno è il solito punto $z=1$ e l'altro è situato lungo il semiasse reale negativo.

Tale curva chiusa $L^{(h)}$ racchiude internamente la solita circonferenza $|z|=1$ e ha in comune con questa il solo punto $z=1$. Detto $E^{(h)}$ il campo limitato avente per frontiera $L^{(h)}$ si ha che, al solito, il dominio circolare $|z| \leq 1$ ò contenuto nel campo $E^{(h)}$ eccetto, s'intende, il panto $z=1$ che sta lungo la frontiera $L^{(h)}$ di $E^{(h)}$.

La curva $L^{(h)}$ è, s'intende, della solita natura, d'avere cioè una sola intersezione con ogni semiretta uscente da $z=0$.

Per tutto quanto si è fatto in $a$ ) si vede che

Il campo $E^{(h)} \dot{e}$ il campo di sommabilità, pel metodo $U_{n}^{(h)}$, nel caso della

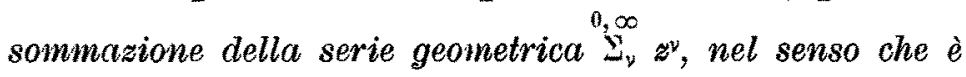

$$
\lim _{n \rightarrow \infty} D_{n}^{\langle h\rangle}(z)=\lim _{n \rightarrow \infty} \sum_{k^{k}}^{0, \frac{n}{2^{n}}} a_{n, 2^{h} \cdot \mu^{k}} \cdot z^{\mu}=\frac{1}{1-z},
$$

uniformemente in ogni dominio $T$ appartenente al campo $E^{(h)}$.

$\left.b^{\prime}\right)$ In generale assegnato un elemento $F(z) \cong \Sigma_{\nu}^{0} u_{v} \cdot z_{y}$, a raggio di convergenza $\neq 0$, il polinomio $U_{n}^{\{h\rangle}(z)$ relativo al procedimento sopra esposto è

$$
U_{n}^{\langle h\rangle}(z)=\stackrel{0, \frac{n}{2^{h}}}{\Sigma_{\mu}^{h}} a_{n, 2^{h} \cdot \mu} \cdot u_{\mu} \cdot z^{i}
$$


e per tale metodo sussiste la proposizione seguente (estensione di quella precisata in $b$ ) pel caso particolare di $h=1$ ).

Ferme restando le notazioni impiegate in b) e se con $E_{F^{\prime}}^{(h)}$ s'indica il campo parte comune a tutti $i$ campi $\alpha \cdot E^{(h)}$, si ha che in ogni dominio $T$ appartenente al campo $E_{F}^{\langle h\rangle} \grave{e}$ uniformemente

$$
\lim _{n \rightarrow \infty} U_{n}^{(h)}(z)=\lim _{n \rightarrow \infty} \Sigma_{\mu,}^{0, \frac{n}{2^{h}}} a_{n, 2^{h} \cdot \mu} \cdot u_{\mu} \cdot z^{\mu}=F(z),
$$

ove $F(z)$ è il ramo monodromo di funzione olomorfa ottenuta col prolungamento analitico dell'elemento $\sum_{v}^{0} u_{\psi} \cdot z^{v}$ entro la relativa stella rettilinea di centro $z=0$.

È bene evidente che in tali problemi l'intero positivo $h$ ha il ruolo di vero e proprio ordine di sommazione e che $E_{F}^{(h)}$ sorpassa il cerchio di convergenza di $\Sigma_{\nu} u_{\nu} \cdot z^{\nu}$ in ogni punto non singolare della circonferenza di convergenza e ciò per ogni valore che si assuma per l'intero positivo $h$.

Osservazione. - Come appare ben evidente i metodi $U_{n}^{(h)}$ offrono fra l'altro il notevole vantaggio di rendere estremamente semplice la struttura del relativo polinomio di sommazione $U_{n}^{(h)}$.

E altresì bene evidente che risultati del tutto analoghi come vantaggio pratico si otterrebbero se sempre in riferimento all'iniziale procedimento lineare di Toeplitz $U_{n}=\sum_{\nu}^{n, n} a_{n, \nu} \cdot u_{\nu}$, si facesse sempre uso della relazione

$$
\frac{1}{2 \xi}\left[\frac{1}{1-\xi}-\frac{1}{1+\xi}\right]=\frac{1}{1-\xi^{2}},
$$

al posto di quella $\frac{1}{2}\left[\frac{1}{1-\xi}+\frac{1}{1+\xi}\right]=\frac{1}{1-\xi^{2}}$ impiegata nel corso di questo lavoro, e iterando $h$ volte il procedimento che se ne trarrebbe in modo perfettamente analogo a quello qui indicato. Senza vieppiù insistere si può ancora rilevare che si ritroverebbero quali campi di sommabilità i suliti $\dot{E}_{F}^{\langle h\rangle}$.

Tracce di analogo indirizzo (per il solo valore $h=1$ ) e usando la relazione $\frac{1}{1-\xi} \cdot \frac{1}{1+\xi}=\frac{1}{1-\xi^{3}}$ si trovano in una precedente categoria di lavori (C. BirindeliI, Una generalizzazione del metodo di sommazione del De La Vallée Poussin per le serie, nella teoria del prolungamento analitico, «R. Isti. tuto Lombardo di Scienze e Lettere», vol. LXIV, fasc. VI, X, (1931); ecc....). La iterazione del procedimento per $h$ volte di seguito porta ad un procedimento di sommazione avente sempre il campo $E_{F}^{(h)}$ come campo di sommabilità. Ma è ben evidente che, con l'eseguire i prodotti $U_{n}(\xi) \cdot U_{n}(-\xi)$ ecc...., i coefficenti $a_{n, v}$ vengono, e più volte, ad essere moltiplicati fra di loro cosicchè la struttura del finale polinomio di sommazione viene ad apparire, specie al crescere di $h$, vieppiù estremamente complicata. Mancherebbe quindi il vantaggio che si riscontra invece nei procedimenti $U_{n}^{(h)}$ qui introdotti, d'essere ciò̀ algoritmicamente estremamente semplici. 
In tal senso, l'attuale lavoro ha quindi, per così dire, un significato anche di rettifica in relazione ai risultati inerenti ai citati lavori precedenti $\left(^{3}\right)$.

6. Ai metodi $(f, g)$ di Gronwall $\left(^{3}\right)$ aventi reali i coefficienti $a_{n, y}$ è agevole applicare oltre alle argomentazioni del n. 5, anche altre utilissime, specie se impiegate nel problema generale del prolungamento analitico di un elemento di funzione olomorfa entro la stella rettilinea.

Assegnata una qualunque serie numerica, $\Sigma_{v} \Sigma_{v}$, è chiaro che il metodo $(f, g)$ le può essere applicato $h$ volte consecutivamente e ben si comprende come tale iterazione del metodo $(f, g)$ viene a costituire un nuovo comples

(3) Le argomentazioni svolte al $\mathrm{n} .5$ sono marcatamente notevoli quando è possibile conoscere l'equazione della frontiera $L$ del campo $D$. Per una assai estesa categoria di metodi lineari [T. H. Gronwalt, Summation of series and conformal mapping, * Annals of Mathematics ", second series, vol. 33, 1932] la teoria generale permette in ogni caso di determinare l'equazione della curva $L$. Conviene pereiò richiamare qui per sommi capi la definizione di tali metodi:

1) Sia $z=f(w)$ olomorfa per $|w| \leq 1$, eccetto $w=1$, in modo che $z=f(w)$ rappresenti $|w|<1$ biunivocamente in un campo aperto $H$ interno a $|z|<1$ in modo ehe $z=1$ e $z=0$ corrispondano a $w=1$ e $w=0$ e che la funzione inversa oltre d'esser olomorfa in $H$ sia pur tale lungo la frontiera di $H$, eccetto al più per $z=1$, ed ammetta lo sviluppo

$$
1-w=(1-z)^{\lambda} \cdot \varphi(z)
$$

con $\lambda \geq 1, \varphi(z)$ olomorfa. in $H$ e lungo il contorno, $\varphi(1)>0, \varphi(0)=1$.

2) Un'altra funzione $g(w)$, rappresentabile per $|w|<1$ con la $g(w)=\frac{0, \infty}{\Sigma_{n}} b_{n} w^{n}, b_{0} \neq 0$, $g(w) \neq 0$ per $|w|<1$, abbia l'espressione $g(w)=(1-w)-\alpha+\gamma(w), x>0$, con $\gamma(w)$ olomorfa per $|w| \leq 1$. Data una qualunque serie $\Sigma_{\nu} u_{\nu}$, si trova che le espréssioni $U_{n}$ ricavate dalla identità

$$
\stackrel{0, \infty}{\Sigma_{y}} u_{\nu} z^{y}=\frac{1}{g(v)} \stackrel{0, \infty}{\Sigma_{n}} b_{n} U_{n} w^{n}
$$

sono polinomi del tipo

$$
\left.U_{n}=\stackrel{0, n}{\Sigma_{y}} a_{n, \nu} \cdot u_{\nu} \quad \text { (coi coefficienti } a_{n, y} \text {. indipendenti dalle } u_{v}\right)
$$

e se $\lambda>1$ costituiscono mediante la successione $\left\{U_{n}\right\}$ un procedimento di sommazione lineare $0, \infty$

di Toeplitz per una serie $\Sigma_{y} u_{\nu}$ dimodoche il limite di $U_{n}$, per $n \rightarrow \infty$, quando esiste, da la somma generalizata, della serie assegnata, secondo il metodo $(f, g)$ di Gronwall ora riassunto.

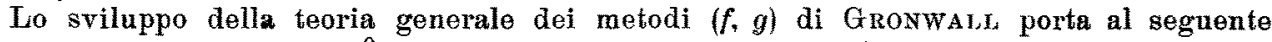
risultato: La serie geometrica $\Sigma_{\nu} z^{\nu}$ è sommabile, con somma $\frac{1}{1-z}$, con un metodo $(f, g)$, se $z$ appartiene al campo limitato $D$ avente per frontiera la linea $L$ di equazione $z=\frac{1}{f\left(e^{i} \varphi\right)}$, $-\pi \leq \varphi \leq \pi$, e in ogni dominio $T \subset D$ la convergenza di $U_{n}(\varepsilon)$ verso $\frac{1}{1-z}$, per $n \rightarrow \infty, \dot{e}$ sempre uniforme; Esternamente a $D$ non si ha convergenga per la successione $U_{4}(z)$. La teoria svolta al n. 5 può così svilupparsi compiutamonte se sono reali tutti i coefficienti $a_{n, y}$ del metodo $(f, g)$ che s'impiega. 
sivo procedimento di sommazione di GronwaLL che applicato direttamente alla serie assegnata $\stackrel{0, \infty}{\Sigma_{\nu}} u_{v}$ trasforma le somme parziali $\stackrel{0, n}{\Sigma_{v}} u_{v}$ della serie nei definitivi polinomi $U_{n}^{(h)}=\Sigma_{\nu}^{0, n} a_{n, \nu}^{(h)} \cdot u_{\nu}$.

Ben si comprende che la determinazione dei coefficienti $a_{n, v}^{(h)}$ del polinomio

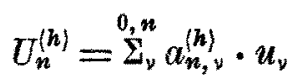

in funzione di quelli $a_{n, v}$ relativi al polinomio $U_{n}$ competente al metodo $(f, g)$ ¿̀ sempre più complicata aigoritmicamente (man mano che $h$ assume valori sempre piu grandi).

Conviene dunque, onde evitare tali inconvenienti, vedere almeno in un caso notevole come si possa ricavare direttamente il polinumio $V_{n}^{(h)}=\sum_{\nu}^{0, n} a_{n, \nu}^{(h)} \cdot u_{y}$ da quello $U_{n}={\stackrel{0, n}{\Sigma}, a_{n, v} \cdot u_{y}}$ senza occuparsi del passaggio dai valori $a_{n, v}$ a quelli $a_{n, y}^{\langle h\rangle}$.

A tale scopo conviene premettere le seguenti

Osservazione 1. - Uno dei teoremi fondamentali della teoria generale dei metodi $(f, g)$ è il seguente

《assegnati due distinti metodi $(f, g),\left(f_{1}, g_{1}\right)$ per cui sia $\lambda<\lambda_{1}, H \supset H_{1}$, allora ogni serie sommabile col metodo $(f, g)$ risulta pure sommabile con l'altro $\left(f_{1}, g_{1}\right)$ e verso la stessa somma generalizzata. Inoltre le serie, di funzioni, uniformemente sommabili col primo metodo, lo sono pure col secondo».

Tale risultato generale sussiste ogni volta che $\lambda<\lambda_{1}$ e se $H_{4}$ \& interno ad $H$, fatta astrazione, s'intende, del comune punto $z=1$ ehe sta lungo le due frontiere di $H$ e $H_{1}$.

OSSERVAZIONE 2: - Diamo qui un semplice procedimento utile per ogni dato metodo $(f, g)$ e che serve a ricavare la espressione di $U_{n}$ in funzione

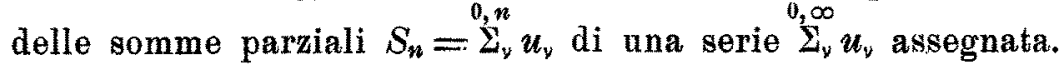

Si parte da

$$
z=f(w)=\stackrel{1, \infty}{\Sigma_{m}} d_{m} \cdot w^{m}, \quad \text { poichè è } d_{0}=0,
$$

onde procurarsi nel modo che segue gli sviluppi in serie di potenze di $w$ per le varie espressioni $z^{\nu}=[f(w)]^{\nu}$ che compaiono nel primo membro della identità $\left({ }^{*}\right)$ di $\left(^{3}\right)$.

Dalla formula di LeIBNITz risulta

$$
D^{r}[f(w)]^{\nu}=\sum_{k_{1}+k_{2}+\ldots+k_{\nu}=r} r ! \frac{f_{\left(k_{1}\right)}^{\left(k_{2}\right)}}{k_{1} !} \cdot \frac{f_{(w)}^{\left(k_{2}\right)}}{k_{2} !} \ldots \frac{f_{(w)}^{\left(k_{\nu}\right)}}{k_{\nu} !}
$$

e questa nel caso particolare di $v=0$ si scrive

$$
D^{r}[f(0)]^{\nu}=\underset{k_{1}+k_{2}+\ldots+k_{y}=r}{\Sigma} r ! d_{k_{1}} \cdot d_{k_{2}} \ldots d_{k_{y}}
$$


e qui è inteso che nessuno degli interi non negativi $k_{1}, \ldots, k_{y}$ possa assumere il valore 0 poichè $f^{(0)}(0)=f(0)=0$.

Segue che

$$
z^{\nu}=[f(w)]^{\nu}=\Sigma_{m}^{\nu, \infty} w_{k_{1}+k_{2}+\ldots+k_{v}=m}^{\Sigma} d_{k_{1}} \cdot d_{k_{2} \ldots} \ldots d_{k_{y}}
$$

e la uguaglianza costituità dalla identità $\left(^{*}\right)$ si può scrivere

$$
\begin{aligned}
& \left\{S_{0}+\Sigma_{y}^{1, \infty}\left(S_{y}-S_{\nu-1}\right) \stackrel{\nu, \infty}{\Sigma_{m}} \dot{w}^{m} \underset{k_{1}+k_{2}+\ldots+k_{y}=m}{\Sigma} d_{k_{1}} \cdot d_{k_{2}} \ldots d_{k_{\nu}}\right\} \cdot\left\{\stackrel{0, \infty}{\Sigma_{\nu} b_{\nu} \cdot w^{\nu}}\right\}= \\
& =\stackrel{0}{\infty}, \infty_{n} b_{n} \cdot U_{n} \cdot w^{n} \text {. }
\end{aligned}
$$

Da tale identità si determinano le espressioni $U_{n}$ applicando il principio d'identità per le serie di potenze.

OSSERVAZione 3. - Un metodo di semplice struttura dovuto a $\mathrm{N}$. OBRECHKOFF (e che s'identifica con il particolare metodo di Gronwall ( 1 - $(1-$ $\left.-w)^{\frac{1}{2}},(1-w)^{-\frac{1}{2}}\right)$ come segnalò in seguito il Prof. G. AscokI $)$ è notevole per l'utilità che può dare nelle argomentazioni scopo di questo numero.

In relazione ad una serie numerica $\Sigma_{\nu}^{0, \infty} u_{v}$, posto $f(w)=f_{\lambda}(w)=1-(1-w)^{\frac{1}{\lambda}}$, $g_{1}(w)=(1-w)^{-i}$, ̀̀ più opportuno sostituire alle iterazioni d'un generico metodo $(f, g)$ l'applicazione del procedimento $\left(1-(1-w)^{\frac{1}{\lambda}},(1-w)^{-1}\right) \equiv\left(f_{\lambda}(w)\right.$, $g_{4}(w)$, analogo a quello sopra citato dell' OBREOHKOFF, con il fare poi crescere indefinitamente il valore del parametro $\lambda$ che viene ad assumere il ruolo d'un ordine di sommazione sostituente quello $h$ d'iterazione, per $h$ volte consecutivamente, d'un arbitrario $(f, g)$,

La funzione inversa della $z=f_{\lambda}(w)=1-(1-w)^{\frac{1}{\lambda}}$ \&े $w=1-(1-z)^{\lambda}$. Per esser quindi $1-w=(1-z)^{\lambda}$ è agevole dimostrare che il campo $H_{\lambda}$, di punti $z$, trasformato di quello $|w|<1$, appartiene sempre al campo $|z|<1$ quando

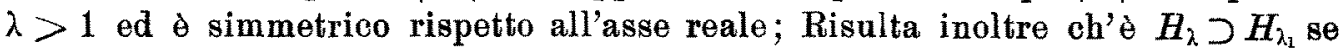
$\lambda<\lambda_{1}$ e in modo tale che il campo $H_{\lambda}$ tende al segmento 01 dell' asse reale quando $\lambda \rightarrow+\infty$.

Per il Teorema della "Osservazione 1 » il procedimento $\left(f_{\lambda}(w), g_{1}(w)\right)$ diventa sempre più potente al crescere di $\lambda$ e il campo di sommabilità $\left({ }^{3}\right)$ della serie geometrica e verso la somma generalizzata $\frac{1}{1-z}$ eे il campo $D_{\lambda}$ limitato avente per frontiera la linea $L_{\lambda}$, simmetrica rispetto all'asse reale e sempre contenente il campo $|z|<1$, di equazione $z=\frac{1}{1-\left(1-e^{i \varphi}\right)^{\frac{1}{\lambda}}}$. $-\pi \leq \varphi \leq \pi$, ove $L_{\lambda}$ è la trasformata, mediante $z^{\prime}=\frac{1}{z}$, della frontiera del campo $H_{\lambda}$. 
Tale campo dị sommabilità $D_{\lambda}$ della serie geometrica, col procedimento $\left(f_{\lambda}, g_{1}\right)$, e verso la somma $\frac{1}{1-z}$, ha per limite il piano complesso tagliato da 1 a $+\infty$ lungo il semiasse reale positivo quando $\lambda \rightarrow+\infty$, vale a dire l'intera stella rettilinea per l'elemento di funzione olomorfa $\Sigma_{\nu} z^{\nu}$ e col cen. tro $z=0$.

Se è assegnato un elemento $F(z) \simeq \Sigma_{\nu}^{0, \infty} u_{y} z^{\nu}$ di funzione olomorfa, con raggio di convergenza $\neq 0$, e se al solito $g^{\prime}$ indica con $\alpha$ il primo punto singolare incontrato quando partendo $\mathrm{da} 0$ si fa il prolungamento analitico rettilineo dell'elemento $F(z)$ lungo qualsiasi semiretta spiccata da 0 , si ha, come conseguenza di quanto si è or detto, il seguente Teorema:

Se con $D_{F}^{(\lambda)} s^{\prime}$ indica il campo parte comune a tutti $i$ campi $\alpha \cdot D_{\lambda}$, si ha che in ogni dominio $T$ appartenente al campo $D_{F}^{(\lambda)} \grave{e ̀ ~ u n i f o r m e m e n t e ~}$

$$
\lim _{n \rightarrow \infty} U_{n}^{(\lambda)}(z)=F(z)
$$

ove $F(z)$ è il ramo monodromo di funzione olomorfa ottenuta col prolungamento analitico dell'elemento $\stackrel{0_{1} \infty}{\Sigma_{y}} u_{y} \cdot z^{\nu}$ entro la relativa stella rettilinea di centro $z=0$. Al crescere di $\lambda$ il campo $D_{F}^{\langle\lambda\rangle}$ di sommabilità, relativo al metodo $\left(f_{\lambda}, g_{1}\right)$ si allarga indefinitamente invadendo l'intera stella rettilinea di centro $z=0$.

Se quindi $z$ è un arbitrario valore interno alla stella rettilinea di centro $z=0$, è possibile assegnare in corrispondenza $a \approx$ un valore di $\lambda$ tale che $D_{F}^{(\lambda)}$ contenga $z e$, di conseguenza, con tale valore di $\lambda \grave{e} \lim _{n \rightarrow \infty} U_{n}^{(\lambda)}(z)=F(z)$. In tal modo l'impiego del procedimento $\left(f_{\lambda}, g_{1}\right)$ nel problema $\overrightarrow{d e l}^{\infty}$ prolungamento analitico d'un elemento di funzione olomorfa $F(z) \cong \Sigma_{v}^{0, \infty} u_{v} z^{\nu}$ equivale all'impiego, per lo stesso scopo, del classico procedimento di Mittag-Leffler.

Resta il problema relativo alla valutazione del polinomio $U_{n}^{(\lambda)}(z)$ pel me. todo $\left(f_{\lambda}, g_{1}\right)$ mediante le somme parziali $S_{n}(z)=\sum_{\nu}^{0, n} u_{\nu} z^{\nu}$ della serie 0 , se si vuole, mediante i termini $u_{z} z^{\nu}$ della serie ${ }^{0, \infty} u_{v} z^{\nu}$ stessa.

Per una serie numerica $\Sigma_{y}^{0, \infty} u_{\vartheta}$ la legge di formazione degli $U_{n}^{\langle\lambda\rangle}$ è, per la $(*)$, riducibile alla

$$
\Sigma u_{y} \cdot\left(1-(1-w)^{\frac{1}{2}}\right)^{v}=(1-w) \cdot \Sigma U_{n}^{(\lambda)} \cdot w^{n}, \quad \text { essendo } \quad b_{n}=1,
$$

e questa si può scrivere

$$
u_{0}+\sum_{\nu}^{1, \infty} u_{v} \cdot\left(1-(1-w)^{\frac{1}{\lambda}}\right)^{\nu}=U_{0}^{(\lambda)}+\Sigma_{n}^{1, \infty}\left(U_{n}^{(\lambda)}-U_{n-1}^{\langle\lambda\rangle}\right) \cdot w^{n} .
$$

D'altra parte

$$
z=f_{\lambda}(w)=1-(1-w)^{\frac{1}{\lambda}}=1-1-\Sigma_{s}^{1, \infty}\left(\frac{1}{\lambda}\right) \cdot(-1)^{s} \cdot w^{s}=\Sigma_{s}^{1, \infty}(-1)^{s+1} \cdot\left(\frac{1}{\lambda}\right) \cdot w^{s}
$$


sicchè nella generale identità (**) si deve porre, nel caso particolare che ci occupa,

$$
d_{s}=(-1)^{s+1} \cdot\left(\frac{1}{\lambda}\right)
$$

Dopo ciò è chiaro che la $\left({ }^{*}\right)$ e la $\left(^{* *}\right)$ portano a considerare l'identita

$$
\begin{gathered}
u_{0}+\sum_{y}^{1, \infty} u_{y} \cdot \sum_{m}^{\Sigma_{m}} w^{m} \sum_{k_{1}+k_{2}+\ldots+k_{y}=m}(-1)^{m+v} \cdot\left(\begin{array}{c}
\frac{1}{\lambda} \\
k_{1}
\end{array}\right)\left(\begin{array}{c}
\frac{1}{\lambda} \\
k_{2}
\end{array}\right) \ldots\left(\begin{array}{c}
\frac{1}{\lambda} \\
k_{y}
\end{array}\right)= \\
=U_{0}^{(\lambda)}+\stackrel{\Sigma}{\Sigma}, \infty_{n}\left(U_{n}^{(\lambda)}-U_{n-1}^{(\lambda)}\right) \cdot w^{n} .
\end{gathered}
$$

Da questa si ottiene che

$$
U_{n}^{(\lambda)}=S_{0}+\Sigma_{i}^{1, n}(-1)^{i} \cdot \stackrel{1, i}{\Sigma}_{\nu}\left(S_{\nu}-S_{\nu-1}\right) \cdot(-1)^{\nu} \cdot \sum_{k_{1}+k_{2}+\ldots+k_{\nu}=i}^{\Sigma}\left(\begin{array}{l}
\frac{1}{\lambda} \\
k_{1}
\end{array}\right)\left(\begin{array}{l}
\frac{1}{\lambda} \\
k_{2}
\end{array}\right) \ldots\left(\begin{array}{l}
\frac{1}{\lambda} \\
k_{\nu}
\end{array}\right) .
$$

Nel caso particolare della serie di potenze si ottiene

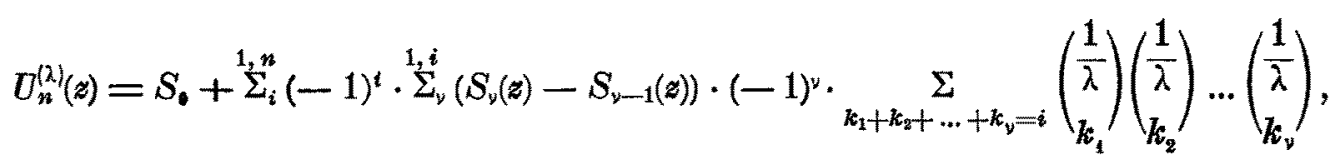

0 , se si vuole,

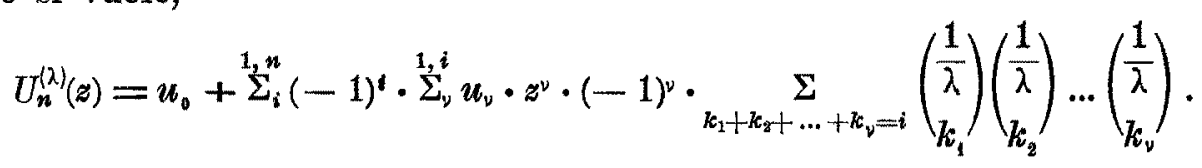

È facile d'altra parte persuadersi che il procedimento $\left(f_{\lambda}, g_{1}\right)$ può interpretarsi come caso particolare di iterazione di un metodo $(f, g)$ eseguita $h$ volte consecutivamente. Ponendo infatti $\lambda^{\prime}=\lambda^{\frac{1}{h}}$ si pensi di iterare per $h$ volte successivamente il particolare procedimento di GronwaLL

$$
\left(1-(1-w)^{\frac{1}{\lambda^{\prime}}}, \quad(1-w)^{-1}\right)
$$

si ha, facendo ad es. il caso di $h=2$, col porre

$$
\begin{gathered}
z=1-\left(1-w^{(1)}\right)^{\frac{1}{\hat{k}^{\prime}}}, \\
w^{(1)}=1-\left(1-w^{(2)}\right)^{\frac{1}{\lambda^{\prime}}},
\end{gathered}
$$

ch'̀े

$$
z=1-\left(1-w^{(2)}\right)^{\left(\frac{1}{\lambda^{\prime}}\right)^{2}}=1-\left(1-w^{(2)}\right)^{\frac{1}{\lambda}}, \text { se } \quad \lambda^{\prime}=\sqrt{\lambda} .
$$


$\dot{\mathrm{E}}$ d'altronde noto dalla teoria generale dei metodi $(f, g)$ che quelli parti colari $\left(f(w),(1-w)^{-1}\right)$ consistono sostanzialmente nella trasformazione della successione $S_{n}$ delle somme parziali della serie $\sum_{\nu}^{0, \infty} u_{v}$ nella successione delle somme parziali $U_{n}$ della serie $\Sigma_{\nu} u_{\nu}{ }^{\prime}$ che si ottiene svolgendo in serie di potenze di $w$ la serie $\Sigma_{y} u_{v} \cdot[f(w)]^{\nu}$ e ponendo poi $w=1$.

¿̀ dunque, per $h=2$, con $\lambda^{\prime}=\sqrt{\lambda}$,

$$
\begin{aligned}
& \left(1-(1-w)^{\frac{1}{\lambda^{\prime}}},\left(1-w^{-1}\right)\left(1-(1-w)^{\frac{1}{)^{\prime}}},(1-w)^{-1}\right) \equiv\right. \\
& \equiv\left(1-(1-w)^{\frac{1}{\lambda^{\prime}}},(1-w)^{-1}\right)^{(2)} \equiv\left(1-(1-w)^{\frac{1}{\lambda}},(1-w)^{-1}\right),
\end{aligned}
$$

$e$, più in generale, per $h$ qualunque,

$$
\left(1-(1-w)^{\frac{1}{\lambda^{\prime}}},(1-w)^{-1}\right)^{(h)} \equiv\left(1-(1-w)^{\frac{1}{\lambda^{\prime}}},(1-w)^{-1}\right), \quad \text { con } \quad \lambda^{\prime}=\sqrt[h]{\lambda}
$$

Venendo infine di nuovo alla questione della iterazione di un generico procedimento $(f, g)$, coi coefficienti $a_{n, y}$ tutti reali, conviene partire dalla $U_{n}^{(1)}=U_{n}=\sum_{v}^{O_{y},} a_{n, \nu} \cdot u_{y}$ per ottenere la formula, esprimente intanto il passaggio diretto dalla successione delle somme parziali $S_{n}^{(0)}=S_{n}$ della $\Sigma_{y} u_{y}$ alla suc. cessione dei polinomi $U_{n}^{\{1}=U_{n}$ inerenti alla sommazione generalizzata della serie stessa col metodo $(f, g)$,

$$
U_{n}^{(1)}=U_{n}=\stackrel{0, n-1}{\Sigma_{\nu}}\left(a_{n, \nu}-a_{n, \nu+1}\right) \cdot S_{y}^{(0)}+a_{n, n} \cdot S_{n}^{(0)} .
$$

La iterazione del metodo $(f, g)$ per $h$ volte successivamente porta alla determinazione della relativa successione $U_{n}^{(h)}$ dei polinomi di approssimazione e la valutazione di $U_{n}^{(h)}$ è riconducibile alle note $S_{n}^{(0)}=U_{n}^{(0)}=S_{n}$ mediante l'applicazione della evidente relazione ricorrente

$$
U_{n}^{(i+1)}=\stackrel{0, n-1}{\Sigma_{y}}\left(a_{n, \nu}-a_{n, v+1}\right) \cdot U_{v}^{\langle i\rangle}+a_{n, n} \cdot U_{n}^{(i)}
$$

facendo successivamente $i=0,1, \ldots, h-1$.

Il campo $H^{(f, g)(1)}$ trasformato di quello $|w|<1$, mediante la $z=f(w)$, è interno al cerchio $|z|<1$, eccetto il punto 1 comune ai contorni $\mathcal{L}_{(f, g)(1)}$ e $|z|=1$ di detti campi. Con l'impiego della stessa trasformazione conforme, ponendo ciò̀

$$
z^{(1)}=f(z)=f[f(w)]=f_{(2)}(w)
$$

il campo $H^{(f, g)^{(1)}}$ di punti $z$, si trasforma in altro, di punti $z^{(1)}, H^{(f, g)^{(2)}} \subset$ $\subset H^{(f, g)^{(1)}} \subset|z|<1$, eccetto il punto 1 comune ai contorni $\mathfrak{S}_{(f, g)^{(2)}}, \mathfrak{S}_{(f, g)(1)}$, $|z|=1$, di detti campi. Seguitando cosi $h$ volte successivamente si ha

$$
H^{(f, g)^{(h)}} \subset H^{(f, g)^{(h-1)}} \subset \ldots \subset H^{(f, g)^{(z)}} \subset H^{(f, g)^{(1)}} \subset|z|<1,
$$




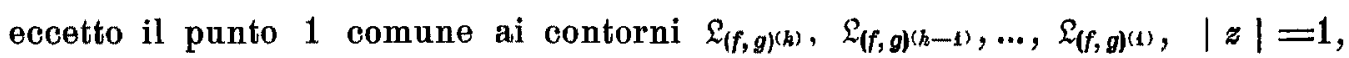
di tali campi. Nella progressiva contrazione del campo $H^{(f, g)^{(k)}}$ che avviene al crescere di $h$ (campo che e sempre simmetrico rispetto all' asse reale) puô darsi che per $h \rightarrow \infty$ il contorno $\mathscr{L}_{(f, g)^{(h)}}$ di $H^{(f, g)^{(h)}}$ tenda a ridursi al segmento 01 dell' asse reale, nel qual caso è possibile adattare tutta la teoria svolta in questo numero pel caso particolare di iterazione del metodo $\left(1-(1-w)^{\frac{1}{\lambda^{\prime}}}\right.$, $\left.(1-w)^{-1}\right)$. Nel caso invece in oui l'insieme limite $\lim _{h \rightarrow \infty} H^{(f, g)^{(k)}}$ non si riduca al detto segmento 01 dell'asse reale, la ripetizione di tutte le argomentazioni precedenti avrebbe minor rilievo sopratutto per il fatto che i risultati che si potrebbero ottenere nel problema del prolungamento analitico rettilineo di un elemento di funzione olomorfa non permetterebbero la sommazione della serie di potenze in tutta l'intera stella rettilinea, il che è invece del tutto realizzabile nel precedente caso. 